\title{
Understanding holmium laser enucleation of the prostate (HoLEP) recovery: Assessing patient expectations and understanding
}

\author{
Mark A. Assmus, MD'; Matthew S. Lee, MD'; Tim Large, MD'; Amy E. Krambeck, MD² \\ Indiana University School of Medicine, Department of Urology, Indianapolis, IN, United States; ${ }^{2}$ Northwestern University, Department of Urology, Chicago, II, United States
}

Cite as: Assmus MA, Lee MS, Large T, et al. Understanding holmium laser enucleation of the prostate (HoLEP) recovery: Assessing patient expectations and understanding. Can Urol Assoc J 2022;16(1):E25-32. http://dx.doi.org/10.5489/cuaj.7328

Published online August 26, 2021

\section{Abstract}

Introduction: Although holmium laser enucleation of the prostate (HoLEP) is a highly effective surgery, there is a variable recovery period where patients may experience hematuria, dysuria, or urinary incontinence (UI). Despite preoperative consultation, there is a paucity of literature examining the effectiveness of physicianpatient communication in preparing the patient for the postoperative recovery period. We sought to examine recovery expectations as a patient-reported outcome (PRO) metric for HoLEP.

Methods: With institutional review board approval, we queried our electronic medical record and retrospective clinical registry to identify 50 consecutive patients that underwent HoLEP from November 2019 to March 2020 by two endourologists. Patients were provided questionnaires via Twistle ${ }^{\odot} \geq 6$ months postoperatively. Patient demographics and perioperative course was examined in the context of responses. Our primary objective was determining whether patients felt they had a reasonable understanding of the recovery process.

Results: We observed a 92\% (46/50) response rate, with an average patient age of 69.4 years (range 55-88). Overall, 91.3\% (42/46) felt they had a reasonable understanding of the recovery. Additionally, $97.8 \%$ (45/46) were aware of temporary UI, with $87 \%$ having $\geq 1$ episodes of UI after catheter removal. We found $47.8 \%(22 / 46)$ of patients expected UI to resolve within 30 days, while $8.6 \%$ expected $>90$ days of UI. All patients were aware of the risk of hematuria, with $93.5 \%$ (43/46) expecting resolution within 30 days (<7 days: $47.8 \%$; $7-14$ days: $28.3 \%$; $15-30$ days: $17.4 \%$ ).

Conclusions: Although surgical technique continues to improve HoLEP, ensuring adequate physician-patient communication to optimize expectations is crucial. We report patient understanding of HoLEP recovery and areas for future improvement.

\section{Introduction}

Bladder outlet obstruction (BOO) secondary to benign prostatic hyperplasia $(\mathrm{BPH})$ is an extremely common disease, with close to $50 \%$ of adult males experiencing moderate to severe lower urinary tract symptoms (LUTS) by the eighth decade of life. ${ }^{1}$ Longitudinal studies and placebo arms from large pharmacological studies have shown that $3.5-10 \%$ of men ultimately proceed to require surgical intervention for $\mathrm{BPH}$ management. ${ }^{1}$ While there are various surgical options, holmium laser enucleation of the prostate (HoLEP) is a guidelinerecommended, durable treatment for all prostate gland sizes.

Although excellent durable outcomes following HoLEP are well-established, there is a variable period of recovery in which patients may experience hematuria, dysuria, or transient urinary incontinence (tUI), which may be stress, urge, or mixed in nature. Fortunately, these symptoms are temporary for the vast majority, with 10 -year post-HoLEP outcomes of stress urinary incontinence (SUI), urgency urinary incontinence (UUI), and reoperation rates of $1 \%$, $0.5 \%$, and $0.7 \%$, respectively. ${ }^{2,3}$ Despite significant preoperative counselling, postoperative feedback from our patients revealed that there may be room for improved physicianpatient communication about the recovery period and where to find reliable resources.

Given the potential information gap identified in our patient population, we sought to formally evaluate and better understand our patient expectations and understanding of post-HoLEP recovery. Overall, there is a paucity of research aimed at patient-perceived expectations of the post-HoLEP recovery period, particularly with respect to LUTS. Most studies exploring patient perceptions focus specifically on the final outcomes of retrograde ejaculation, erectile function, sexual satisfaction, or post-HoLEP outcomes beyond the transient recovery period. . $^{4-8}$

Along with limited research on patient perceptions of post-HoLEP recovery is an abundance of accurate and inaccurate information available on the Internet, which can make it challenging for the general public to determine source credibility. ${ }^{9}$ Taken together, it is crucial to ensure physicianpatient communication of postoperative expectations is clear and that reliable sources of additional information are available, particularly when patients may experience a temporary impairment to quality of life (QoL) domains. Our primary objective was to assess patient understanding and expecta- 
tions of the post-HoLEP recovery period. Our secondary objective was to identify areas where physician-patient communication of postoperative expectations could be improved and how to ensure patients had access to reliable resources.

\section{Methods}

A 10-part patient questionnaire (Fig. 1) was created with input from volunteer patients, clinic urology nurses, medical assistants, urology residents, fellows, and staff urologists. Following institutional review board approval, this self-developed, nonvalidated questionnaire was then administered to 50 adult ( $\geq 18$ years old) patients physically and cognitively capable of completing the questionnaire who had undergone HoLEP by two endourologists at our center between November 2019 and March 2020. Branch logic was used to provide individual questions relevant to respondents' understanding and expectations of the post-HoLEP recovery, as evidenced by their specific responses. The questionnaires were administered to patients within their postoperative followup course $\geq 6$ months after surgery using their mobile Twistle ${ }^{\odot}$ application. The Twistle application enabled text message communication of patient-reported surveys and care concerns between the patient and endourology care team. ${ }^{10}$ Completion of the questionnaire was voluntary, and patients were informed that their decision to participate would have no direct effect on their care, particularly since all patients had completed their surgical intervention and their standard first postoperative followup appointments.

Patient demographics, questionnaire results, and perioperative clinical outcomes were added to an encrypted anonymized REDCap database. As our primary outcome, we assessed whether patients reported having a reasonable understanding of the post-HoLEP recovery process. Secondary outcomes included patient understanding, expectation, and perceptions of post-HoLEP recovery, including dysuria, UI, hematuria, and patient-reported ways to improve physicianpatient communication of reliable resources. Continuous variables were expressed as mean and range, while proportions were used for categorical variables. SPSS statistical program ${ }^{11}$ was used for the statistical analysis, including heteroscedastic two-tailed T-test, Chi-squared test, and Wilcoxon rank sum test for non-parametric variables. Statistical significance was set at $\mathrm{p}<0.05$. This manuscript adheres to the SQUIRE 2.0 standards of publishing quality initiatives in medicine. ${ }^{12}$

\section{Results}

\section{Characteristics}

We observed a 92\% (46/50) response rate, with average age 69.4 years (range 55-88) and mean preoperative pros- tate size of $91.8 \mathrm{~mL}$ (range 30.7-237.2). Patient demographics and perioperative characteristics are outlined in Table 1 . Over one-quarter $(28.3 \%)$ of patients experienced some degree of preoperative $\mathrm{UI}$, with four patients $(8.7 \%)$ reporting wearing liners or incontinence products prior to HoLEP. Patients that used dual antiplatelet medication or therapeutic anticoagulation (6/46, 13\%) were referred to their prescribing physician for preoperative medical approval and medication planning. None of these patients required perioperative bridging and they were all able to hold antiplatelet or anticoagulants perioperatively.

There were no intraoperative complications and no Clavien-Dindo $\geq 3 \mathrm{~B}$ complications within 90 days. All patients had their postoperative catheter removed successfully within 24 hours of surgery, with two patients initially failing same-day trial of void (TOV) but passing successfully on postoperative day 1 (POD1). One patient had Gleason grade group (GGG) 1 prostate cancer detected on prostate biopsy prior to HoLEP and there were two new cases of prostate cancer detected on HoLEP pathology (one GGG1 in $<1 \%$ of tissue, one GGG2 in $5 \%$ of tissue). The two patients with GGG1 prostate cancer elected for continued surveillance with prostate-specific antigen (PSA) and digital rectal exam post-HoLEP. The patient with GGG2 detected on HoLEP pathology had a negative preoperative prostate biopsy and baseline PSA of 4.26 and following his HoLEP, elected for referral for consideration of treatment.

\section{Objective outcomes}

Table 2 shows a comparison of baseline preoperative serum laboratory values (hemoglobin, creatinine, PSA) and validated patient reported QoL questionnaires (American Urological Association symptom score [AUASS], QoL score, BPH index, Michigan Severity Incontinence (MISI) score, Sexual Health Inventory for Men [SHIM], Generalized Anxiety Disorder-7 [GAD-7], Male Sexual Health Questionnaire Ejaculatory Dysfunction Short Form [EjD]) to three-month postoperative outcomes. HoLEP provided a significant reduction in serum PSA (5.05 vs. 0.55, $\mathrm{p}<0.001)$ and improvement in AUASS (24.5 vs. 5.7, $\mathrm{p}<0.001)$, QoL score (4.5 vs. 2.0, $\mathrm{p}<0.001), \mathrm{BPH}$ index (7.7 vs 2.1, $\mathrm{p}<0.001)$, and MISI (9.1 vs $5.3, \mathrm{p}=0.037)$. Objective improvement in uroflow parameters was identified post-HoLEP (peak urinary flow [Qmax] 6.1 vs. $15.5, p=0.020$ ). There was no difference between preoperative and post-HoLEP GAD-7, EjD, or SHIM scores.

\section{Understanding}

When asked, "Looking back, do you feel you had a reasonable understanding of the overall healing process after this type of surgery?", 91.3\% (42/46) selected yes. Of the four patients who responded that they did not have a reason- 


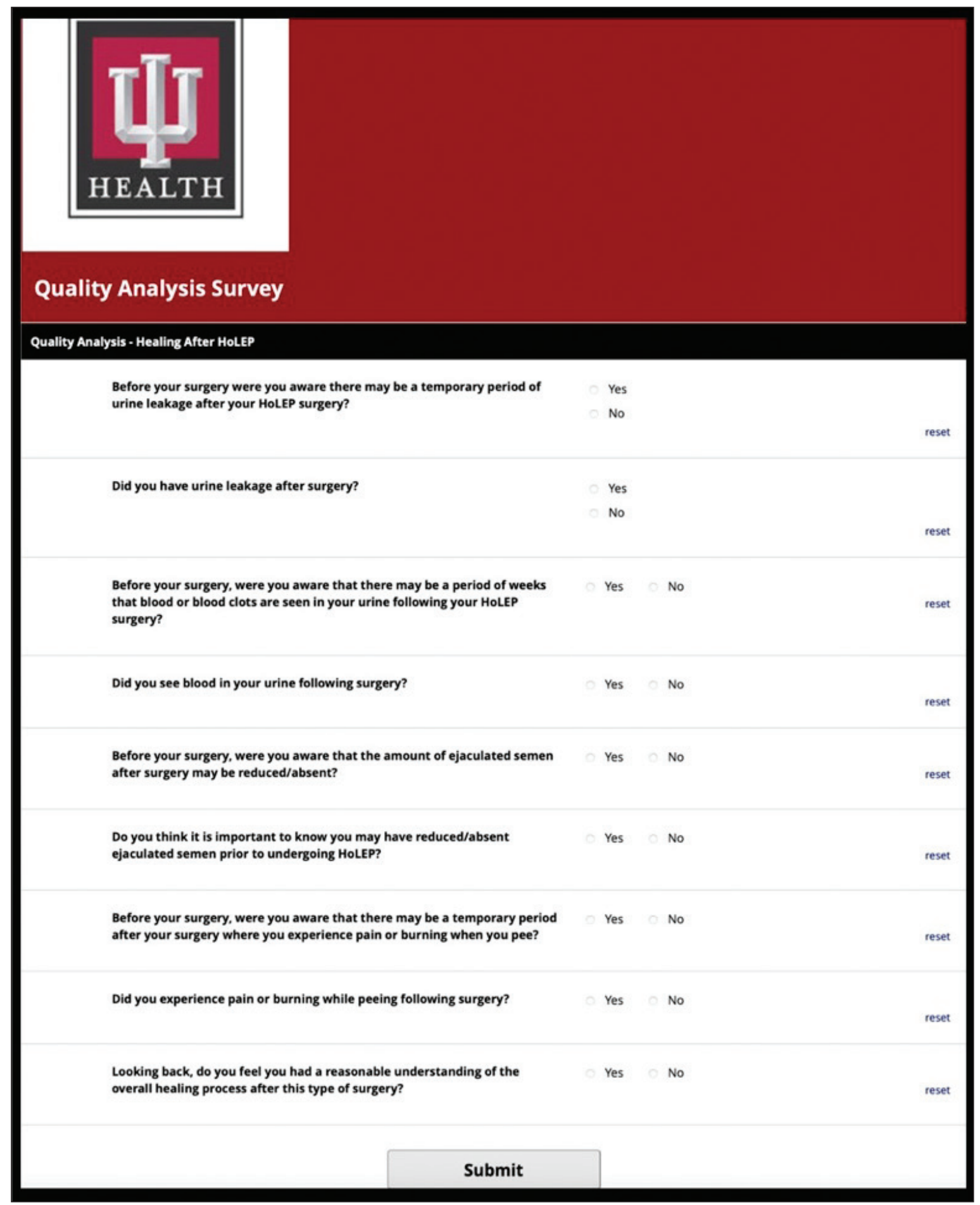

Fig. 1. General template of our 10-part patient questionnaire regarding their expectation and understanding of the post-holmium laser enucleation of the prostate (HoLEP) recovery period. Branch logic questions based on respondent answers were populated to tailor survey to individual patient experience and expectations.

able understanding, 75\% responded yes to being aware of all four recovery period symptoms queried (UI, hematuria, dysuria, and retrograde ejaculation). Of the respondents who felt they did not have a reasonable understanding, only one patient reported not being aware there may be both retrograde ejaculation and dysuria. Although unaware of possible retrograde ejaculation and dysuria, this patient experienced no dysuria postoperatively and reported that he did not feel 


\begin{tabular}{|c|c|c|}
\hline Variable & Mean & Range \\
\hline Age (years) & 69.4 & $55-88$ \\
\hline $\mathrm{BMI}$ & 29.5 & $22.2-43.4$ \\
\hline ASA score & 2.5 & $2-4$ \\
\hline Enucleation time (min) & 45.2 & 14-102 \\
\hline Morcellation time (min) & 7.8 & $1-20$ \\
\hline $\begin{array}{l}\text { Total procedure time including ancillary } \\
\text { procedures (min) }\end{array}$ & 87.5 & $41-164$ \\
\hline Energy used (kJ) & 112.9 & $39.7-251.6$ \\
\hline \multirow[t]{2}{*}{ Intraoperative pathology specimen weight (g) } & 68.0 & 7-164 \\
\hline & $\mathbf{n}$ & $\%$ \\
\hline Preoperative urinary incontinence & 13 & $28.3 \%$ \\
\hline Rarely, uses no protection & 9 & $19.6 \%$ \\
\hline Often, wears liners or incontinence product & 4 & $8.7 \%$ \\
\hline Moses 2.0 mode used & 39 & $84.8 \%$ \\
\hline Perineal urethrostomy used & 0 & $0 \%$ \\
\hline Bladder neck incised & 7 & $15.2 \%$ \\
\hline Concurrent urolithiasis surgery & 6 & $13.0 \%$ \\
\hline Unilateral ureteroscopy & 0 & $0 \%$ \\
\hline Bilateral ureteroscopy & 1 & $2.2 \%$ \\
\hline Cystolitholapaxy & 5 & $10.8 \%$ \\
\hline Urinary retention at time of HoLEP & 11 & $23.9 \%$ \\
\hline Indwelling urethral catheter & 6 & $13.0 \%$ \\
\hline Clean intermittent catheterization & 5 & $10.9 \%$ \\
\hline History of urinary retention & 19 & $41.3 \%$ \\
\hline Therapeutic antiplatelet/anticoagulation use & 6 & $13.0 \%$ \\
\hline Antiplatelet (clopidogrel) & 3 & $6.5 \%$ \\
\hline Anticoagulant (warfarin) & 3 & $6.5 \%$ \\
\hline Prostate cancer detected on pathology & 3 & $6.5 \%$ \\
\hline Gleason grade group I & 2 & $4.3 \%$ \\
\hline Gleason grade group II & 1 & $2.2 \%$ \\
\hline
\end{tabular}

ASA: American Society of Anesthesiology; BMI: body mass index; HoLEP: holmium laser enucleation of the prostate.

knowledge of retrograde ejaculation was important prior to surgery.

Patients who reported not having a reasonable understanding of the healing process had shorter expected duration of UI compared to patients who reported a reasonable understanding $(p=0.024)$. There was no difference between expected duration of postoperative hematuria between patients who reported a reasonable understanding of recovery vs. those that did not ( $p>0.05)$. There was no difference in reported understanding between primary surgeon $(p>0.05)$. Comparing objective validated symptom score results between patients who reported a reasonable understanding vs. those that did not identified no difference in pre- or post-HoLEP AUASS, MISI severity, MISI bother, EjD, or GAD-7 scores (all $p>0.05$ ). The patients reporting that they did not have a reasonable understanding of HoLEP recovery had worse preop QoL (5 vs. 4.5, $\mathrm{p}=0.011)$ and $\mathrm{BPH}$ impact (10 vs. 7.5, $\mathrm{p}<0.001)$ scores along with better postoperative SHIM scores (22.5 vs. 13.2, $\mathrm{p}=0.0082)$ compared to those with reasonable understanding.
Table 2. Comparison of preoperative serum PSA, validated symptom scores (AUASS, QoL, BPH Index, SHIM, MISI, GAD-7, EjD), postvoid residuals, and urinary flow to 3-month followup

\begin{tabular}{lccc}
\hline Variable & $\begin{array}{c}\text { Baseline } \\
\text { preoperative } \\
\text { mean (range) }\end{array}$ & $\begin{array}{c}\text { 3-month } \\
\text { postoperative } \\
\text { mean (range) }\end{array}$ & p \\
\hline Serum PSA, ng/mL & $5.05(0.096-16.5)$ & $0.55(0.00-2.10)$ & $<0.001$ \\
Serum hemoglobin, & $14.30(10.4-17.7)$ & $14.03(11.3-16.4)$ & 0.60 \\
g/dL & $1.06(0.67-2.39)$ & $1.09(0.72-2.10)$ & 0.73 \\
Serum creatinine, & & & \\
mg/dL & $24.5(13-35)$ & $5.7(0-19)$ & $<0.001$ \\
AUASS & $4.5(2-6)$ & $2.0(0-7)$ & $<0.001$ \\
QoL score & $7.7(0-13)$ & $2.1(0-8)$ & $<0.001$ \\
BPH index & $9.1(0-24)$ & $5.3(0-18)$ & 0.037 \\
MISI & $11.5(0-25)$ & $14.1(1-25)$ & 0.33 \\
SHIM & $3.7(0-21)$ & $2.8(0-14)$ & 0.54 \\
GAD-7 & $7.4(0-20)$ & $6.9(0-16)$ & 0.72 \\
EjD & $166.7(38-750)$ & $22.2(0-74)$ & 0.0013 \\
Postvoid residual & & & \\
(mL) & $6.1(2.6-14)$ & $15.5(8.3-26.6)$ & 0.020 \\
Omax (mL/s) & $3.3(1.1-10.1)$ & $8.1(3.4-16.8)$ & 0.016 \\
Qavg (mL/s) &
\end{tabular}

AUASS: American Urological Association symptom score; BPH: benign prostatic hyperplasia; EjD: Male Sexual Health Questionnaire Ejaculatory Dysfunction Short Form; GAD-7: Generalized Anxiety Disorder-7; MISI: Michigan Severity Incontinence score; PSA: prostate-specific antigen; Qavg: average urinary flow; Omax: peak urinary flow; QoL: quality of life; SHIM: Sexual Health Inventory for Men.

\section{Urinary incontinence}

Overall, $97.8 \%$ (45/46) were aware there may be temporary UI, with $87 \%$ of respondents having $\geq 1$ episode of UI after catheter removal. With regards to symptom resolution, $47.8 \%(22 / 46)$ of patients expected their UI to resolve within 30 days, while $8.6 \%$ expected $>90$ days of UI (Fig. 2 ). The patient unaware of the risk of UI reported the degree of not knowing to be $1 / 10$ on a 10-point Likert scale $(1=$ no bother). All patients that were continent at three-month followup reported achieving adequate continence prior to their expected duration of postoperative UI. Seven patients had any $\mathrm{UI}$ at three-month followup and one patient had ongoing $\mathrm{UI}$ at six-month followup.

\section{Hematuria}

All patients (46/46) were aware of the risk of transient hematuria after HoLEP, with $93.5 \%$ (43/46) expecting resolution within 30 days or less. Overall distribution of postoperative hematuria duration was reported as $<7$ days in $47.8 \%, 7-14$ days in $28.3 \%$, and $15-30$ days in $17.4 \% ; 36 / 46$ (78.3\%) of patients reported seeing gross hematuria following catheter removal after HoLEP. When postoperative hematuria was observed, 33/36 thought, "I'm not worried," while two wondered, "Is this normal?" and one patient felt, "Something 


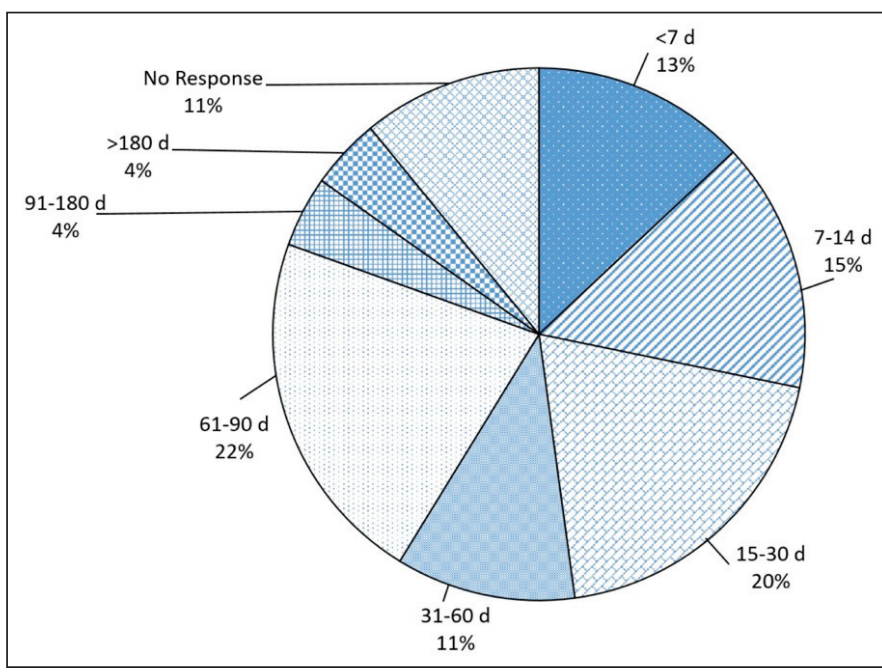

Fig. 2. Patient-reported expectation of urinary continence duration (days) after holmium laser enucleation of the prostate (HoLEP) surgery.

went wrong in my surgery." The patient who felt something went wrong reported an expected duration of postoperative hematuria up to 14 days, with his hematuria resolution occurring on POD 23.

\section{Retrograde ejaculation}

Despite preoperative counselling, 10.9\% (5/46) of patients reported that they were not aware ejaculate volume may change postoperatively. Of these men, $4 / 5$ (80\%) reported that information about retrograde ejaculation is important information to understand prior to proceeding with HoLEP. There was no significant difference in preoperative or threemonth postoperative SHIM or EjD scores between patients who reported being aware of possible retrograde ejaculation vs. those that were not (all p>0.05). Overall, 41/46 (89.1\%) felt that it is important to understand possible changes to ejaculation prior to proceeding with HoLEP.

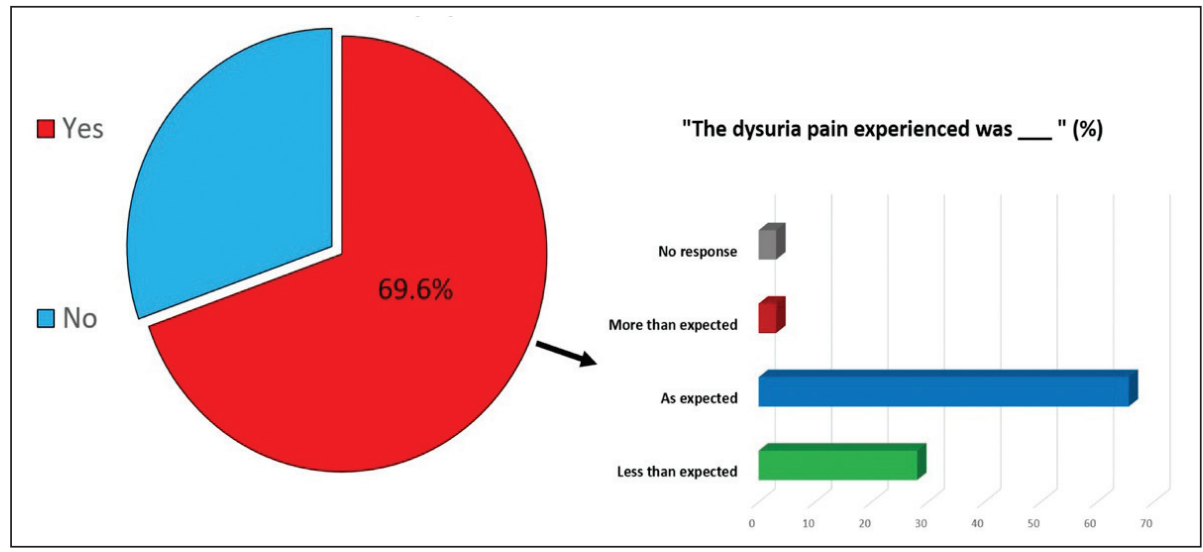

Fig. 3. Patient-reported postoperative dysuria and perception of post-holmium laser enucleation of the prostate (HoLEP) dysuria severity.

\section{Dysuria}

After catheter removal, 32/46 (69.6\%) experienced any dysuria, with $65.6 \%$ reporting "as expected," $28.1 \%$ "less painful," and 3.1\% "more painful." (Fig. 3).

\section{Improving communication}

Of the 46 respondents, 44 provided feedback on ways to improve physician-patient communication. The three most common patient-reported ways to improve communication were: $27.3 \%$ handout, $18.2 \%$ spend more time, and $18.2 \%$ explain to family member/friend. Only $9.1 \%$ felt communication could improve with a HoLEP website (Fig. 4). Patients who felt recovery understanding could be improved with recommended online resources or HoLEP website were younger than patients who did not feel that would improve understanding (63.9 years vs. 69.7 years, $\mathrm{p}<0.001)$.

\section{Discussion}

Over $90 \%$ of patients reported a reasonable understanding of the post-HoLEP recovery process, with the majority expecting potential common postoperative symptoms and signs (e.g., dysuria, hematuria, UI). However, our quality assessment of patient's perceptions also highlights that understanding common postoperative symptom duration is important to the patients surveyed and we plan to incorporate these finding into future quality improvement for preoperative counselling in our practices. The identification of patient perceptions and information that they deem important in the post-HoLEP recovery period is crucial to educate effectively at the time of consultation. With patient travel distance in mind and given the unique, transient QoL-impairing recovery period that may not be familiar to local urologists, ensuring successful physician-patient communication may also help improve patient satisfaction, while reducing office phone calls and postoperative patient stress.

A noticeable unanticipated finding from our study was that most patients do not believe that a website or online resource supported by the endourology team would be beneficial for further information. This may represent a technology-averse cohort of patients. In fact, those patients that supported more online resources were significantly younger than the average study age. One study examining BPH information-seeking behaviors of 479 men before and after a variety of $\mathrm{BPH}$ surgeries found that overall patients felt it was easy to find information on $\mathrm{BPH}$ and its treats- 


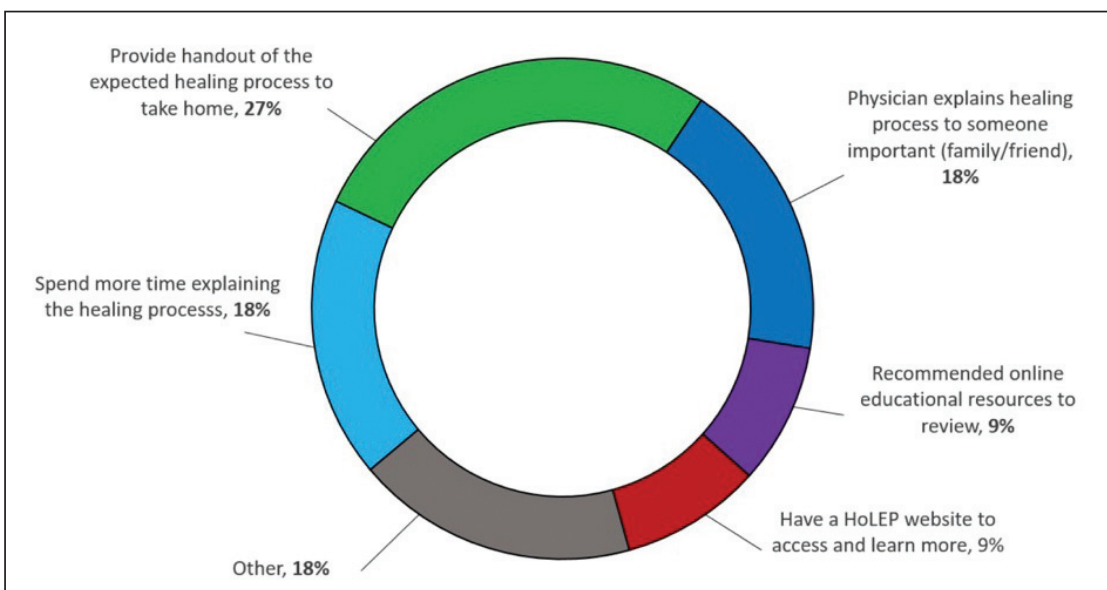

Fig. 4. Patient-reported ways to improve physician-patient communication of the post-holmium laser enucleation of the prostate (HoLEP) recovery period. patients still were not aware that they would develop retrograde ejaculation after HoLEP. Such a communication gap indicates some patients require a different style of counselling to ensure proper education.

We also identified significant variation in patient understanding and expectations of postoperative UI, particularly with respect to duration. We believe this is important, as patients who reported that they did not have a reasonable understanding of the recovery period expected significantly shorter postoperative UI duration compared to patients who had a reasonable understanding. Despite multiple studies showing improvement in $\mathrm{UI}$ rates up to 12 months from primary or secondary HoLEP, a definitive tool to predict and counsel ment options online. ${ }^{9}$ Although the inclusion for their study was men $\geq 50$ years old, they did not report the average or range of age of respondents, and this may represent a younger cohort. Interestingly, close to $75 \%$ of men in their study reported that communicating with other patients experiencing the same BPH symptoms or postoperative concerns was not important and $\mathrm{BPH}$ support groups are rarely used, which may point towards the sensitive nature of urinary symptoms. This highlights a further reliance on the physician-patient communication of accurate and patientdeemed important factors of the recovery period.

Further supporting the importance of communicating patient-defined goals and expectations, 170 patients were examined across validated surveys before and after HoLEP at one, three, six, and 12 months. ${ }^{13}$ Prior to HoLEP, patients determined key self-assessed goals of the treatment and were subsequently asked to provide self-assessed goal achievement (SAGA) and overall satisfaction scores. The authors found that SAGA responses improved with time from surgery, correlated to overall treatment satisfaction, and varied significantly between patients. This highlights that simply examining single traditional objective measures (e.g., International Prostate Symptom Score [IPSS]) uniformly across all patients may provide an inaccurate evaluation of satisfaction.

Our findings reinforce the literature regarding patientdefined importance of ejaculatory changes post-BPH surgery and further support that, irrespective of baseline or postoperative SHIM or EjD scores, a specific cohort of patients may be identifiable for having significant concern regarding retrograde ejaculation through adequate preoperative communication. ${ }^{5,6}$ At time of preoperative counselling, patients are counselled by the fellow and then the staff surgeon regarding the development of retrograde ejaculation after HoLEP. They then visit with a nurse, who provides them written literature on retrograde ejaculation. However, despite this extensive preoperative counselling, $10 \%$ of our patients on their anticipated UI timeline has not yet been developed. ${ }^{3,14}$ One study looking at overall patient reported satisfaction after 331 HoLEPS found $91.8 \%$ were satisfied..$^{15}$ Importantly, of the patients that were dissatisfied, one of the most common reasons was transient postoperative UI. Of note, dissatisfied patients in that study had higher postoperative IPSS scores at six months (11.7 vs. $6.8, p<0.001) .{ }^{15}$ Although distinct from overall satisfaction, our study found no difference in preoperative or postoperative AUASS in patients reporting a reasonable understanding vs. those that did not.

At consultation for consideration of HoLEP at our center, patients are explained risks, benefits, side effects, and typical perioperative course by the endourology fellow and primary surgeon. Subsequently, the surgery consent process and further questions are reviewed with the patient by the primary surgeon. After these two interactions, the patient interacts with our surgical nurse, who reviews the standardized perioperative course and provides patients a uniform handout containing HoLEP recovery information. Despite three levels of counselling, in our study, the highest patient-reported way to improve physician-patient communication of post-HoLEP recovery was to provide a handout for patients to take home. Other studies aiming to improve physician-patient knowledge transfer have shown improved patient understanding and information retention when the handout was physically provided to patients by the surgeon and this represents an area for future evaluation. ${ }^{16}$

Beyond seeking to improve physician-patient communication itself, there are additional potential benefits of improving patient understanding. Often, patients undergoing elective HoLEP are searching for QoL improvement and a recovery period with new or worsened LUTS can lead to stress, frustration, and regret between their initial goals and perceived early surgical outcomes. Although not changing the occurrence of the transient recovery symptoms, aiming 
to reduce avoidable patient stress with increased understanding throughout the healing process is a priority for our urology team. Secondly, patient understanding of the recovery is important in early identification of patients that may be veering off the standard pathway. Although there are planned regular postoperative visits with their surgical team, there remain periods of time where patients are managing the recovery symptoms independently. Empowering patients throughout this period so that they can reach out to their care team and receive reassurance or further evaluation is crucial. This early notification could avoid worse complications or need for additional treatments. Finally, there may be a reduction in patient-initiated postoperative office phone calls for regular symptoms of the HoLEP recovery, potentially improving the use of ancillary urology team members' time. Further studies are required to objectively determine these benefits of improved physician-patient communication for HoLEP recovery.

A strength of our study is correlation of responses to validated pre- and postoperative questionnaires (AUASS, SHIM, MISI, GAD-7). One limitation is excluding patients who were physically or cognitively unable to complete the survey. This cohort of excluded patients and healthcare power of attorney represent an area that requires further investigation. Additionally, we do not have specific data on all comorbidities that may impair ejaculatory or sexual function, such as degree of diabetic control or testosterone deficiency syndrome. Also, patient-reported retrograde ejaculation and erectile function, or dysfunction, is a subjective reported measure. Beyond use of the questionnaire and additional validated QoL surveys, we did not perform objective verification of these patient-reported outcomes (e.g., post-ejaculation urinalysis). It is important to acknowledge that we used a non-validated patient questionnaire. However, the objective of our study was to explore the under-reported patient perceptions and own expectations of the recovery process itself and not to explore previously well-studied objective HoLEP outcomes or validated surveys. Finally, our study was a noncomparative design with a relatively short-term followup.

Building upon our initial evaluation of patient perceptions of the HoLEP recovery, we look forward to developing quality improvement interventions to bridge the gap in physician-patient communication to ensure patients understand the expected and potential timeline of the post-HoLEP recovery period.

\section{Conclusions}

Although surgical technique and technologies continue to improve objective outcomes of HoLEP, ensuring adequate physician-patient communication of the potential recovery period to optimize patient expectations is crucial. We report patient understanding of HoLEP recovery and highlight areas for future improvement.

Competing interests: Dr. Large has been a consultant for Boston Scientific and Lumenis. Dr. Krambeck has been a consultant for Ambu, Boston Scientific, Lumenis, and Virtuoso; and has been on the Data Safety Monitoring Board for Sonomotion. The remaining authors do not report any competing personal or financial interests related to this work.

This paper has been peer-reviewed.

\section{References}

1. Fitzpatrick JM. The natural history of benign prostatic hyperplasia. BJU Int 2006;97:3-6. htrps://doi.org/10.1111/i.1464-410X.2006.06097.x

2. Elmansy HM, Kotb A, Elhilali MM. Holmium laser enucleation of the prostate: Long-term durability of clinical outcomes and complication rates during 10 years of followup. J Urol 2011;186:1972-6. https://doi.org/10.1016/i.juro.2011.06.065

3. Large T, Krambeck AE. Evidence-based outcomes of holmium laser enucleation of the prostate. Curr Opin Urol 2018;28:301-8. https://doi.org/10.1097/MOU.0000000000000498

4. Klett DE, Tyson MD 2nd, Mmeje CO, et al. Patient-reported sexual outcomes after holmium laser enucleation of the prostate: A 3-year followup study. Urology 2014;84:421-6. https://doi.org/10.1016/i. urology.2014.04.042

5. Kim JK, Cho MC, Son H, et al. Patient perception of ejaculatory volume reduction after holmium laser enucleation of the prostate (HoLEP). Urology 2017;99:142-7. https://doi.org/10.1016/i.urology.2016.09.037

6. Gild P, Dahlem R, Pompe RS, et al. Retrograde ejaculation after holmium laser enucleation of the prostate (HoLEP) - impact on sexual function and evaluation of patient bother using validated questionnaires. Andrology 2020;8:1779-86. https://doi.org/10.1111/andr.12887

7. Placer J, Salvador C, Planas J, et al. Effects of holmium laser enucleation of the prostate on sexual function. J Endourol 2015;29:332-9. https://doi.org/10.1089/end.2014.0502

8. Sturch P, Woo HH, McNicholas T, et al. Ejaculatory dysfunction after treatment for lower urinary tract symptoms: Retrograde ejaculation or retrograde thinking? BJU Int 2014;115:186-7. https://doi.org/10.1111/bju. 12868

9. Abdu-Muhsin H, Tyson M, Raghu S, et al. The informed patient: An analysis of information-seeking behavior and surgical outcomes among men with benign prostatic hyperplasia. Am J Mens Health 2017;11:147-53. https://doi.org/10.1177/1557988315617527

10. Twistle. [Mobile Application Software]: 011 Silver Ave SE Albuquerque, New Mexico 87108; 2020 Available at: https://www.twistle.com. Accessed Aug. 26, 2021.

11. Corporation I. IBM SPSS Statistics for Windows. In: Armonk NIC, editor. 27.0 ed. Released 2020.

12. Ogrinc G, Davies L, Goodman D, et al. SQUIRE 2.0 (Standards for Quality Improvement Reporting Excellence): Revised publication guidelines from a detailed consensus process. BMJ Qual Safety 2015;41:474-9. https://doi.org/10.1016/s1553-7250(15)41062-1

13. Cho MC, Kim JK, Ha SB, et al. Self-assessed goal achievement (SAGA) after holmium laser enucleation of the prostate (HoLEP): Association with patients' postoperative satisfaction. PLOS One 2018;13:e203825. hitps://doi.org/10.1371/journal.pone.0203825

14. Jaeger CD, Krambeck AE. Holmium laser enucleation of the prostate for persistent lower urinary tract symptoms after prior benign prostatic hyperplasia surgery. Urology 2013;81:1025-9. https://doi.org/10.1016/i.urology.2013.01.019

15. Lee YJ, Oh SA, Kim SH, et al. Patient satisfaction after holmium laser enucleation of the prostate (HoLEP): A prospective cohort study. PLoS One 2017;12:e0182230. https://doi.org/10.1371//ournal. pone. 0182230

16. Assmus MA, McLarty R, Senthilselvan A, et al. Improving knowledge transfer by using a summative patient handout for cystoscopy. Urol Pract 2020;7:356-61. https://doi.org/10.1097/ UPJ.0000000000000116

Correspondence: Dr. Mark A. Assmus, Indiana University School of Medicine, Department of Urology, Indianapolis, IN, United States; assmus@ualberta.ca 